\title{
Directional Surface Wave Spectra from Point Measurements of Height and Slope
}

\author{
William J. Plant \\ Applied Physics Laboratory, University of Washington, Seattle, Washington \\ MARK A. DONELAN ${ }^{\mathrm{a}}$ \\ Rosenstiel School of Marine and Atmospheric Science, University of Miami, Miami, Florida
}

(Manuscript received 1 August 2019, in final form 29 October 2019)

\begin{abstract}
We describe here a method for recovering directional ocean surface wave spectra obtained from height and slope measurements made over a small area, the iterative deconvolution method (IDM). We show that IDM is a more reliable method for estimating directional wave spectra than more common spectral estimation techniques by comparing it with the widely used maximum entropy method (MEM). IDM is based on the observation that pitch-roll buoys produce directional spectra that are the true spectra convolved with an angular windowing function and are therefore much broader than the true spectra. We test IDM against simulated data and find that it does a better job of retrieving the known input spectra than does MEM, which often produces false double peaks or incorrect angular widths. We compare IDM recoveries to spectra obtained using a nonstandard processing technique, the wavelet directional method (WDM) on data from a compact array of wave staffs on Lake Ontario. We find that IDM produces directional wave spectra very nearly identical to those obtained using WDM, verifying both techniques. Finally, we processed standard NDBC buoy directional spectra and showed that IDM recovers ocean wave spectra that narrow in the Strait of Juan de Fuca and that follow a changing wind in the expected manner. Neither of these phenomena are reliably obtained using MEM due to its tendency to produce false bimodal peaks and peaks that are too narrow.
\end{abstract}

\section{Introduction}

In a seminal paper, Longuet-Higgins et al. (1963, hereafter, LCS) showed that ocean surface height and slope measurements from a pitch-roll buoy could be analyzed in such a way as to produce the first five Fourier angular coefficients of a directional wave spectrum. This truncated Fourier series could be expressed as the true directional spectrum convolved with a nonunique windowing function, which the analyst is free to choose. In particular, if $F(f, \varphi)$ is the true directional surface wave spectrum where $f$ is frequency and $\varphi$ is the direction from which the wave comes measured clockwise from north, then the spectrum obtained from the buoy data $F_{B}(f, \varphi)$ can be written

\footnotetext{
${ }^{\mathrm{a}}$ Deceased.
}

Corresponding author: William J. Plant, plant@apl.washington.edu

$$
\begin{aligned}
F_{B}(f, \varphi) & =\frac{1}{2 \pi} \int F\left(f, \varphi^{\prime}\right) W\left(\varphi-\varphi^{\prime}\right) d \varphi^{\prime} \\
& =\frac{F(f)}{2 \pi} \int \Phi\left(f, \varphi^{\prime}\right) W\left(\varphi-\varphi^{\prime}\right) d \varphi^{\prime}=F(f) D(f, \varphi),
\end{aligned}
$$

where we have broken the directional spectrum into an omnidirectional part $F(f)$, and an angular spreading function $\Phi(f, \varphi)$, whose angular integral is one at every frequency. This equation shows that the spreading function $D$ obtained from the buoy is the convolution of the true spreading function with a windowing function and thus much broader than $\Phi$. We discuss below the nonuniqueness of $W$ and, therefore, of $D ; F(f)$ may be obtained directly from the surface height measurements of the buoy.

We now proceed where many have gone before (LCS; Kuik et al.1988; Earle et al. 1999; Benoit 1994). Let $\gamma(t)$, $s_{n}(t)$, and $s_{e}(t)$ be time series of the surface displacement and the north and east components of slope measured by the buoy. Note that these same physical quantities can 
be obtained by other means, such as arrays of wave staffs. We write the complex Fourier transforms (FFTs) of these quantities as $\tilde{\gamma}(f), \widetilde{s}_{n}$, and $\tilde{s}_{e}$. Then the first five Fourier coefficients of the directional wave spectrum are

$$
\begin{aligned}
& a_{o}=\frac{\left\langle|\tilde{\gamma}|^{2}\right\rangle}{\pi \Delta f}, \\
& a_{1}=\frac{\operatorname{imag}\left(\left\langle\tilde{s}_{n}^{*} \tilde{\gamma}\right\rangle\right)}{k \pi \Delta f}, \\
& b_{1}=\frac{\operatorname{imag}\left(\left\langle\tilde{s}_{e}^{*} \tilde{\gamma}\right\rangle\right)}{k \pi \Delta f}, \\
& a_{2}=\frac{\left\langle\left|\tilde{s}_{n}\right|^{2}\right\rangle-\left\langle\left|\tilde{s}_{e}\right|^{2}\right\rangle}{k^{2} \pi \Delta f}, \\
& b_{2}=\frac{2 \operatorname{real}\left(\left\langle\tilde{s}_{n}^{*} \tilde{s}_{e}\right\rangle\right)}{k^{2} \pi \Delta f},
\end{aligned}
$$

where $k$ is wavenumber, angle brackets indicate an ensemble average, $\Delta f$ is the frequency spacing of the FFT, and the subscripts on the left indicate the harmonic number. We are free to combine these coefficients in any way we want as long as we maintain the harmonic order, thus leading to the nonuniqueness of $W$ (see appendix A). In this paper, we combine these five Fourier coefficients in the same manner as LCS:

$$
\begin{aligned}
F_{B}(f, \theta)= & \frac{a_{o}}{2}+\frac{2}{3}\left(a_{1} \cos \theta+b_{1} \sin \theta\right) \\
& +\frac{1}{6}\left(a_{2} \cos 2 \theta+b_{2} \sin 2 \theta\right),
\end{aligned}
$$

where $\theta$ is the angle toward which the wave propagates measured counterclockwise from east. Here we want to use the NDBC convention with the angle $\varphi$ being the angle from which the wave propagates measured clockwise from north (Steele et al. 1992). The two conventions are related by $\varphi=270^{\circ}-\theta$. Accordingly, we make the following substitutions: $\sin \varphi=-\cos \theta, \cos \varphi=-\sin \theta$, $\cos 2 \varphi=-\cos 2 \theta$, and $\sin 2 \varphi=\sin 2 \theta$ to get

$$
\begin{aligned}
F_{B}(f, \varphi)= & \frac{a_{o}}{2}-\frac{2}{3}\left(a_{1} \sin \varphi+b_{1} \cos \varphi\right) \\
& -\frac{1}{6}\left(a_{2} \cos 2 \varphi-b_{2} \sin 2 \varphi\right),
\end{aligned}
$$

which yields the positive-definite windowing function

$$
W\left(\varphi-\varphi^{\prime}\right)=\frac{8}{3} \cos ^{4} \frac{1}{2}\left(\varphi-\varphi^{\prime}\right) .
$$

In appendix A we discuss the possible windowing functions that can be produced with various combinations of the coefficients in Eq. (2). If we define

$$
\begin{aligned}
& r_{1}=\frac{\sqrt{a_{1}^{2}+b_{1}^{2}}}{a_{o}}, \\
& r_{2}=\frac{\sqrt{a_{2}^{2}+b_{2}^{2}}}{a_{o}}, \\
& a_{1}=-a_{o} r_{1} \sin \varphi_{1}, \quad b_{1}=-a_{o} r_{1} \cos \varphi_{1}, \\
& a_{2}=-a_{o} r_{1} \cos 2 \varphi_{2}, \quad b_{2}=a_{o} r_{1} \sin 2 \varphi_{2},
\end{aligned}
$$

then, referring to Eq. (1), we have

$$
\begin{aligned}
F(f) & =\pi a_{o}, \\
D(f, \varphi) & =\frac{0.5+2 r_{1} \cos \left(\varphi-\varphi_{1}^{\prime}\right) / 3+r_{2} \cos \left[2\left(\varphi-\varphi_{2}^{\prime}\right)\right] / 6}{\pi},
\end{aligned}
$$

where

$$
\begin{aligned}
& \varphi_{1}^{\prime}=\tan ^{-1}\left(\frac{a_{1}}{b_{1}}\right), \\
& \varphi_{2}^{\prime}=\frac{1}{2} \tan ^{-1}\left(-\frac{b_{2}}{a_{2}}\right) .
\end{aligned}
$$

These equations produce two values for each $\varphi_{1}^{\prime}$ and $\varphi_{2}^{\prime}$ that are $180^{\circ}$ apart. The proper ones are chosen based on $\varphi_{1}^{\prime}$ being near the wind direction and $\varphi_{2}^{\prime}$ being near $\varphi_{1}^{\prime}$. The NDBC website gives values of $a_{o}$ (called $\left.c_{11}\right), r_{1}, r_{2}$, $\varphi_{1}^{\prime}$, and $\varphi_{2}^{\prime}$ as functions of frequency (Steele et al. 1992).

\section{The iterative deconvolution method}

These equations are the basis for standard methods for deriving directional wave spectra from heights and slopes measured by buoys or, indeed, measured by any other means spanning small areas. However, the buoy spreading function $D(f, \varphi)$ is the convolution of $W$ and $\Phi$ as given by Eq. (1), and the full width of $W$ at half maximum is $130^{\circ}$. Therefore, if the true spreading function $\Phi(\varphi)$ were unidirectional, that is, a delta function in $\varphi$, the spreading function $D(f, \varphi)$ from the buoy would be $130^{\circ}$ wide at half maximum. For wider $\Phi$, $D(f, \varphi)$ will always be wider than this. Because pitch-roll buoys yield such broad spreading functions, various estimation techniques have been developed over the years to attempt to recover the true spreading function $\Phi(\varphi)$. A dozen of these were summarized and investigated by Benoit (1994). He found that the maximum likelihood method (MLM) and the maximum entropy method (MEM) performed best. Another method, the wavelet directional method (WDM), which requires processing of buoy data in a nonstandard way using wavelets, has been developed recently (Donelan et al. 1996). 

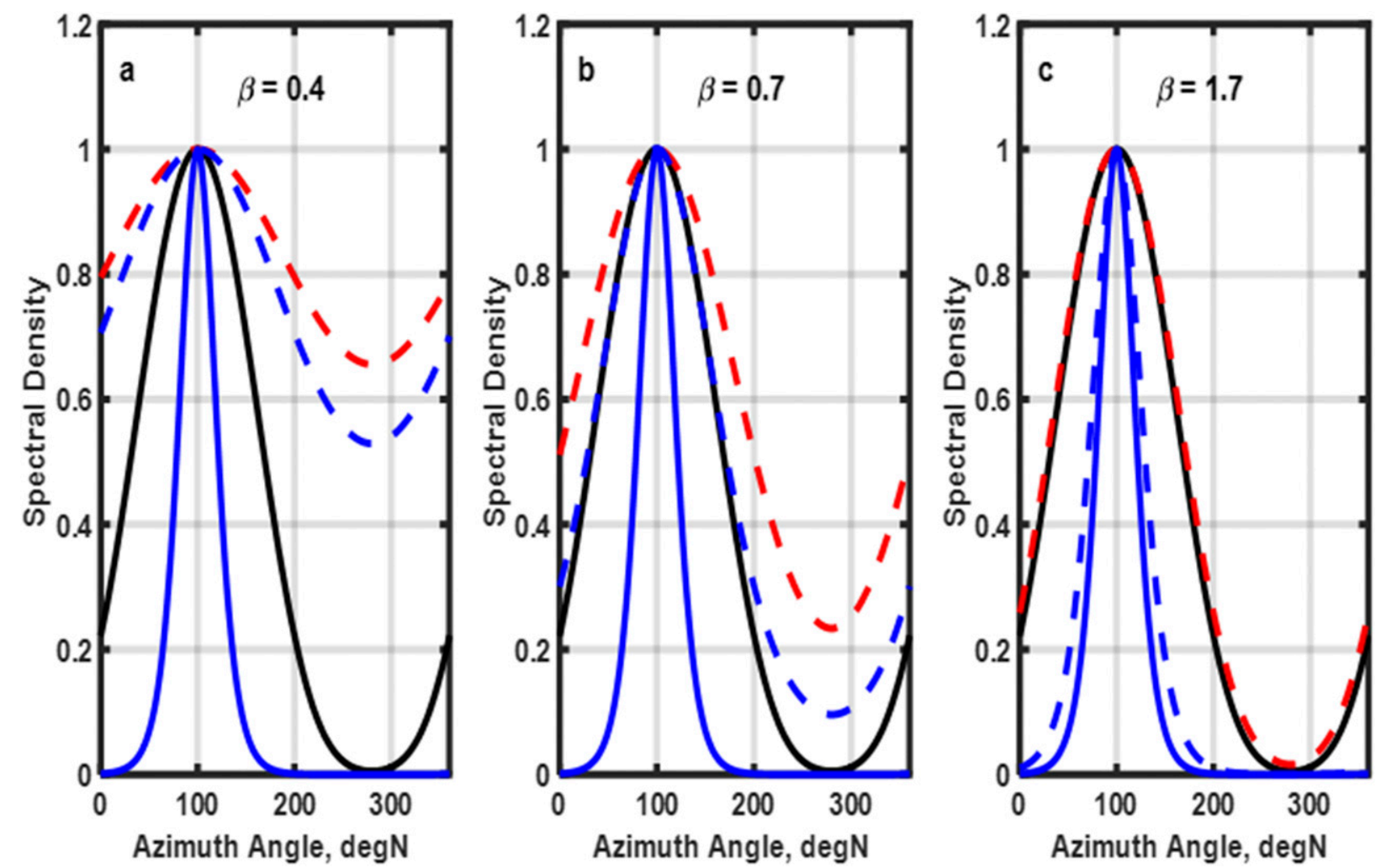

FIG. 1. Iterative deconvolution for a spreading function with a single peak. The solid black curve is $D(f, \varphi)$, the spreading function measured by a buoy or other point measurement. The solid blue curve is the true spreading function $\Phi(f, \varphi)$ that we attempt to recover. Values of $\beta$, the width parameter in Eq. (11), that yield the blue dashed approximation to $\Phi(f, \varphi)$ are given in each panel. The dashed red curve is the convolution of that approximate function with the window function. Since the dashed red curve in (a) does not match the black one, we iterate by increasing the value of $\beta$ to recompute $\Phi(f, \varphi)$ and get the narrower dashed functions shown in (b). Iteration ends when the red dashed curve lies very close to the black curve, as in (c), and the last value of $\beta$ is the recovered width parameter.

WDM has been extensively compared with MLM and MEM (Donelan et al. 2015; Donelan 2017) and generally been found to perform better than the other two, which can over or underpredict simulated spectral widths and can produce spurious peaks. In these studies, WDM produced a spreading function that conformed well to the $\operatorname{sech}^{2}(\beta \varphi)$ spreading function obtained from measurements with a large array of wave gauges (Donelan et al. 1985).

We report here an iterative deconvolution method (IDM) based on Eq. (1), which can use data already processed by the National Data Buoy Center and presented on their website (ndbc.noaa.gov/data/historical/). In this respect, IDM is similar to other methods except WDM. Like Kuik et al. (1988), the present method can be used for the routine analysis of pitchroll buoy data. Whereas Kuik et al. have attempted to specify parameters of the directional spectrum without assuming a model for it, the present method requires a parametric model of the spreading function.
We utilize the form that was first suggested by Donelan et al. (1985). This form is

$$
\Phi(f, \varphi)=\operatorname{sech}^{2}\left\{\beta(f)\left[\varphi-\varphi_{o}(f)\right]\right\} / \int_{-\pi}^{\pi} \operatorname{sech}^{2}[\beta(f) \varphi] d \varphi,
$$

which ensures that $\int_{\varphi_{o}-\pi}^{\varphi_{o}+\pi} \Phi(f, \varphi) d \varphi=1$ and that its maximum occurs in the direction $\varphi_{o}$. We note, however, that this assumption hardly affects the output of the method, which primarily specifies the direction and width of $\Phi(f, \varphi)$. Other shapes for the spreading function can be obtained as a sum of two wave trains. The width parameter $\beta$ and mean direction $\varphi_{o}$ are functions of frequency.

The iteration process is simple if $\Phi$ is unimodal because $\Phi\left(\varphi-\varphi_{o}\right)$ should then be symmetric about $\varphi_{o}$. However, noisy measurements can yield $\varphi_{1}^{\prime}$ and $\varphi_{2}^{\prime}$ of Eqs. (9) and (10) that are slightly different even when they should be equal. In these cases, the spreading function will be 

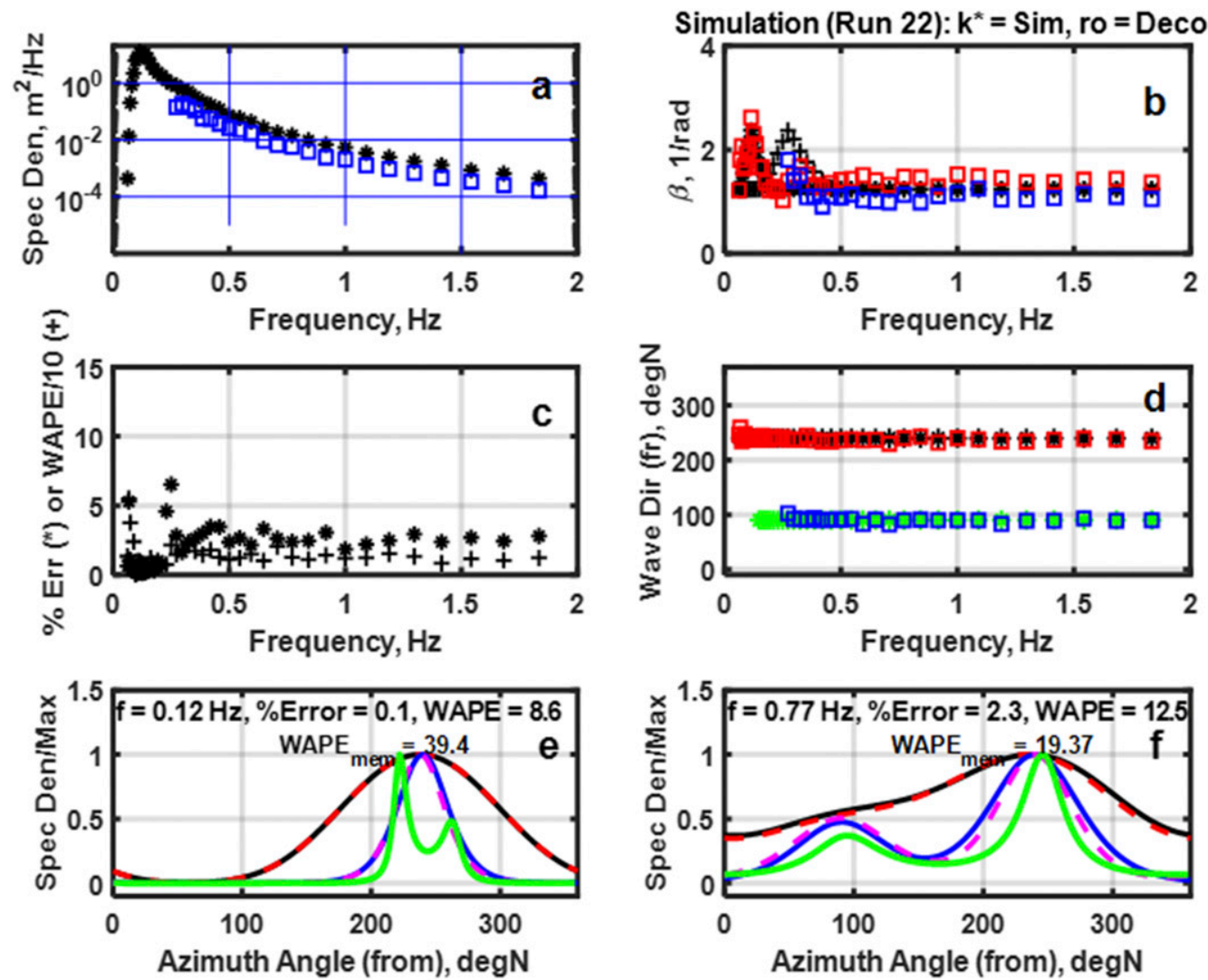

FIG. 2. Comparison of spectral parameters and spreading functions with simulations for input mean wave directions $\varphi_{1}=240^{\circ}$ and $\varphi_{2}=90^{\circ}$. (a) Wave height variance spectrum $F(f)$. Black asterisks are the recovered total spectral density; blue squares are the recovered spectral density of the lower wind speed spectrum $F_{s}(f)$. (b) Width parameters of the wave trains. Input values: black asterisks are $\beta_{1}$, and black plus signs are $\beta_{2}$. IDM recovered values: red squares are $\beta_{1}$, and blue squares are $\beta_{2}$. (c) \%Error (asterisks) and WAPE/10 (plus signs) for IDM. (d) Mean directions of the wave trains. Input directions: black asterisks are $\varphi_{1}$, and green asterisks are $\varphi_{2}$. Recovered directions: blue squares are $\varphi_{1}$, and red squares are $\varphi_{2}$. (e) Spreading functions at frequency $=0.12 \mathrm{~Hz}$. Windowed spreading function $D(f, \varphi)$ : input is solid black, and IDM recovered is dashed red. True spreading function $\Phi(f, \varphi)$ : input is solid blue, IDM recovered is dashed magenta, and MEM recovered is solid green. (f) As in (e), but at a frequency of $0.77 \mathrm{~Hz}$.

unimodal but not symmetric and the method to be described below may produce a second peak. To guard against this possibility we set $\varphi_{1}$ and $\varphi_{2}$ both equal to their mean if they differ by less than $5^{\circ}$. Furthermore, because we need to access $\varphi_{o}$ in a range of $0<\varphi_{o}<2 \pi$ without wrapping $D$ and $\Phi_{1}$, we must ensure that $D$ and $\Phi_{1}$ are periodic by concatenating each over a range $-2 \pi<$ $\varphi_{o}<4 \pi$.

A simple description of the iterative deconvolution method in this case is easily outlined: We begin with a spreading function given by $\Phi_{1}=\operatorname{sech}^{2}\left\{\beta_{1}(f)[\varphi-\right.$ $\left.\left.\varphi_{D}(f)\right]\right\}$, which maximizes to one at $\varphi_{D}$ where $D(f, \varphi)$ is a maximum, and initially use a small value of $\beta_{1}$ (a wide spreading function). The value of $\beta_{1}$ is then iteratively increased until the convolution of $\Phi_{1}$ with $W$ matches the spreading function from the buoy, $D(f, \varphi)$, to within some small, negative value. That is, we continuously increase $\beta_{1}$ until the following condition is first reached (see Fig. 1):

$$
\left.\frac{D(f, \varphi)}{\max (D)}\right|_{1 / 2}-\left.\frac{\Phi_{1}\left(\varphi-\varphi_{D}\right)^{*} W(\varphi)}{\max \left(\Phi_{1}{ }^{*} W\right)}\right|_{1 / 2} \geq-0.001,
$$

where the asterisk $(*)$ denotes convolution and $\left.\cdots\right|_{1 / 2}$ indicates evaluation at the angle $\varphi$, where $D$ is half its maximum value. At this point, we end the iteration and use the final $\beta_{1}$ as the width parameter. We will show below that this procedure yields a far more accurate width for the spreading function than does MEM.

If two wave trains exist at a single frequency, the process becomes considerably more complicated. We document the process in appendix B. In this case, $\Phi=\Phi_{1}+a \Phi_{2}$, where $a(\leq 1)$ determines the relative amplitudes of the 

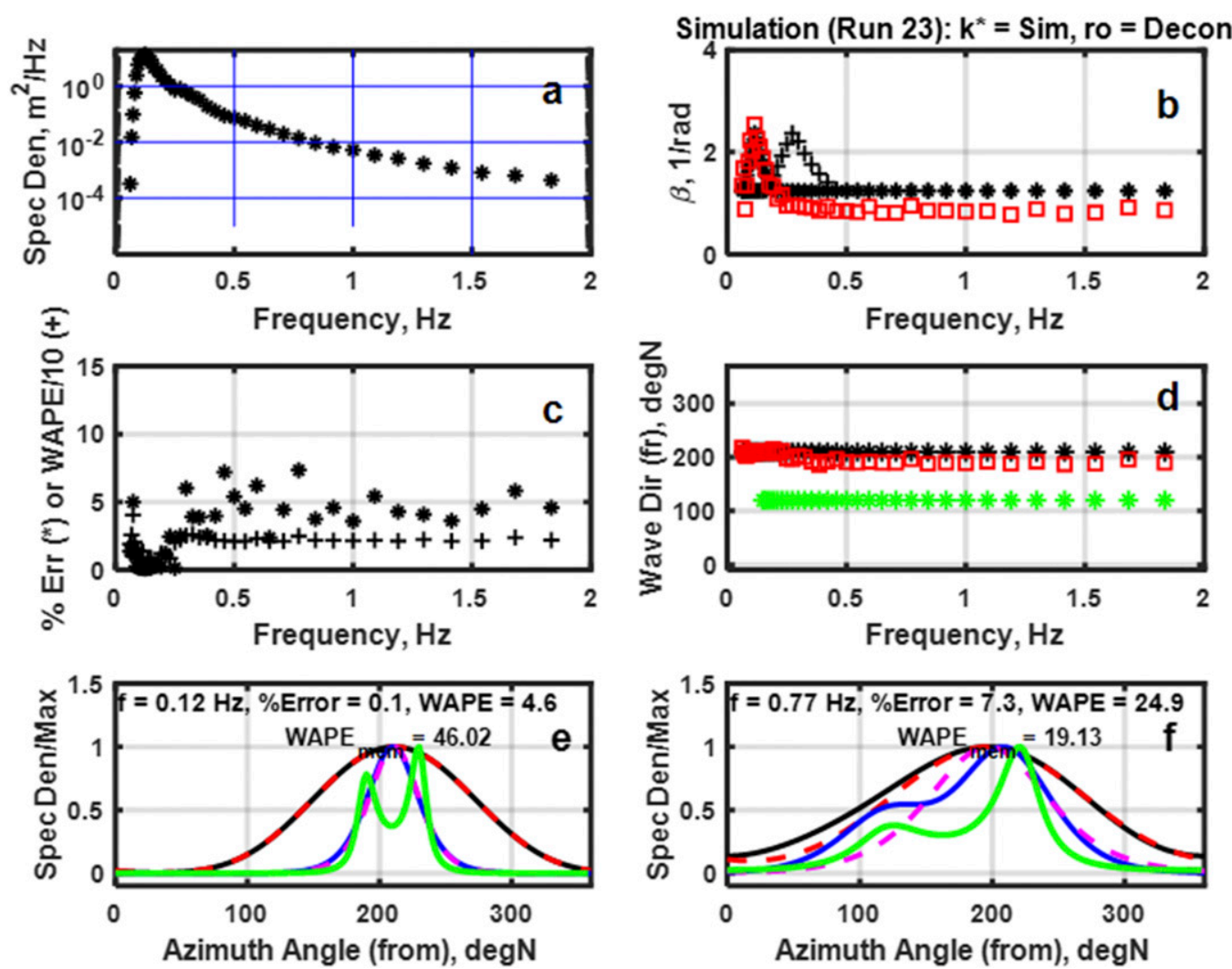

FIG. 3. As in Fig. 2, but for input mean wave directions $\varphi_{1}=210^{\circ}$ and $\varphi_{2}=120^{\circ}$.

two spreading functions and $\Phi_{2}=\operatorname{sech}^{2}\left\{\beta_{2}(f)\left[\varphi-\varphi_{2}(f)\right]\right\}$. Then we have

$$
\begin{aligned}
D(f, \varphi)= & {\left[\Phi_{1}\left(f, \varphi-\varphi_{1}\right) * W(\varphi)\right.} \\
& \left.+a \Phi_{2}\left(f, \varphi-\varphi_{2}\right)^{*} W(\varphi)\right] / \int_{-\pi}^{\pi} \operatorname{Do}(f, \varphi) d \varphi,
\end{aligned}
$$

where the quantity in square brackets here is Do and we must iteratively determine $\beta_{1}, \beta_{2}, \varphi_{1}, \varphi_{2}$, and $a$. We give details of this process in appendix $\mathrm{B}$ where we show that these parameters can be determined if $\left|\varphi_{1}-\varphi_{2}\right|>\sim 130^{\circ}$, the full width at half max of $W$. If this condition is satisfied, then the spreading function is the sum of two sinc ${ }^{2}$ functions, which, of course is not a sinc ${ }^{2}$ function. If the condition is not satisfied, the method simply returns a single, broad $\operatorname{sinc}^{2}$ function whose peak is centered somewhere between $\varphi_{1}$ and $\varphi_{2}$ depending on $a$. We will give examples of these functions below.

\section{Comparison of spreading functions recovered with IDM and MEM with simulations}

To test the IDM and MEM recovery methods, we have artificially produced time series of height and slope from specified spectra, $F_{1}(f), \Phi_{1}(f, \varphi)$, and $F_{2}(f), \Phi_{2}(f, \varphi)$, where subscripts designate the two wave trains. We have followed Donelan et al. (1985) to specify $F_{1}(f)$ at a wind speed of $15 \mathrm{~m} \mathrm{~s}^{-1}$ and a fetch of $500 \mathrm{~km}$ while specifying $F_{2}(f)$ at a wind speed of $8 \mathrm{~m} \mathrm{~s}^{-1}$ and a fetch of $50 \mathrm{~km}$. Spreading functions are given by $\Phi_{i}=\operatorname{sech}^{2}\left\{\beta_{i}(f)[\varphi-\right.$ $\left.\left.\varphi_{i}(f)\right]\right\}, i=1,2$, and $\varphi_{i}$ is varied. Then we write

$$
\begin{aligned}
F(f, \varphi) & =\left[F_{1}(f)+F_{2}(f)\right] \Phi_{t}(f, \varphi), \\
\Phi_{t} & =\frac{F_{1} \Phi_{1}+F_{2} \Phi_{2}}{M},
\end{aligned}
$$

where $M$ is a normalizing factor that gives $\int_{-\pi}^{\pi} \Phi_{t}(\varphi) d \varphi=$ 1. Then the amplitude of the surface displacement due to each Fourier component is given by

$$
A_{j}=\sqrt{\left(a_{r j}^{2}+b_{r j}^{2}\right) F(f) d f},
$$

where $a_{\mathrm{rj}}$ and $b_{\mathrm{rj}}$ are independent, zero mean, unit variance random variables and $j$ runs over the number of frequencies used, $N$. The random phases of the individual Fourier components are $\phi_{j}=\tan ^{-1}\left(a_{r j}^{2} / b_{r j}^{2}\right)$. Wave trains of height and slope are then computed from the following equations: 

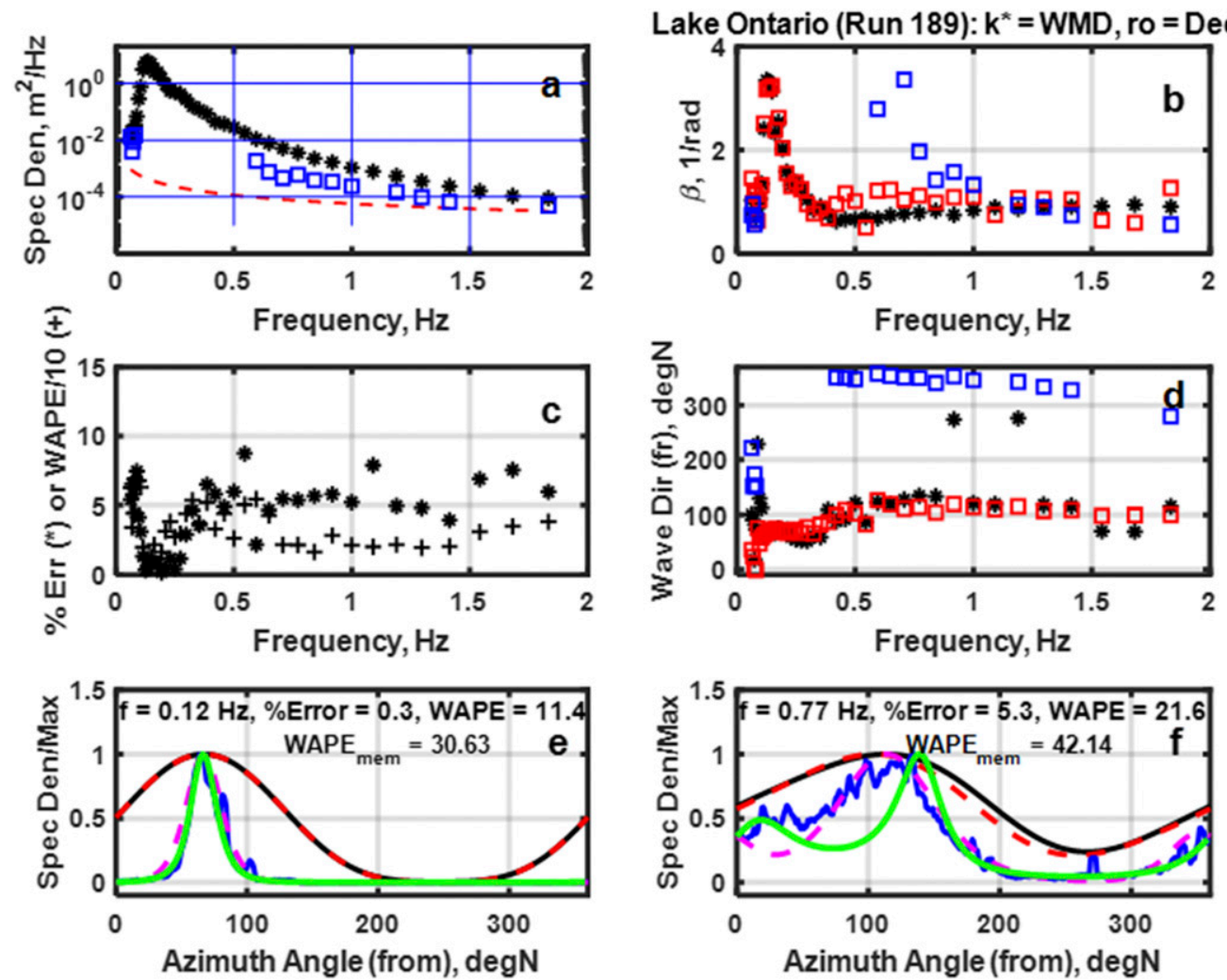

FIG. 4. Spectral parameters and spreading functions recovered by IDM and WDM for Lake Ontario data of run 189 during 1987. Spreading functions recovered by MEM are also shown. (a) Measured wave height variance spectrum $F(f)$. Black asterisks are from wave heights, blue squares are from the weaker spreading function given by IDM. (b) Spreading parameter $\beta$. Black asterisks are from WDM, red squares are $\beta_{1}$ from IDM, and the blue squares are $\beta_{2}$ IDM. The WDM values have been corrected using upwind and crosswind slope variances as outlined by Donelan et al. (1985). (c) \%Error (black asterisks) and WAPE/10 (black plus signs). (d) Mean directions of the wave trains. Black asterisks are from WDM, red squares are for the stronger wave train from IDM, and blue squares are for the weaker wave train from IDM. (e) Spreading functions at a frequency of $0.12 \mathrm{~Hz}$. Windowed spreading function $D(f, \varphi)$ : buoy data are solid black, recovered by IDM is dashed red. True spreading function $\Phi(f, \varphi)$ : estimated by WDM is solid blue, recovered by IDM is dashed magenta, and recovered by MEM is solid green. (f) As in (e), but at a frequency of $0.77 \mathrm{~Hz} . \mathbf{U}_{10}=11.3 \mathrm{~m} \mathrm{~s}^{-1}$ from $84^{\circ} \mathrm{N}$.

$$
\begin{aligned}
\gamma(t) & =\sum_{j=1}^{N} A_{j} \cos \left(\omega_{j} t+\phi_{j}\right), \\
s_{e}(t) & =\sum_{j=1}^{N} k_{j} \sin \varphi A_{j} \sin \left(\omega_{j} t+\phi_{j}\right), \\
s_{n}(t) & =\sum_{j=1}^{N} k_{j} \cos \varphi A_{j} \sin \left(\omega_{j} t+\phi_{j}\right) .
\end{aligned}
$$

These time series are processed as if they were measured data. Results of processing these using IDM and MEM to recover spreading functions are shown in Figs. 2 and 3.

MEM was implemented using the function MEMdirectionalestimator.m written by Paul Jensen of the Naval Postgraduate School in 2003 with slight modifications made by Jim Thomson of the University of Washington Applied Physics Laboratory in 2005.
This function can be obtained from the website GitHub (in the repository jthomson-apluw/SWIFTcodes/Waves). No changes were made to this function by the authors.

Figure 2 shows the results for $\varphi_{1}=240^{\circ}, \varphi_{2}=90^{\circ}$, so the separation of the mean directions of the wave trains is $\Delta \varphi_{12}=\varphi_{1}-\varphi_{2}=150^{\circ}$. This separation exceeds the $130^{\circ}$ width of the window function $W$ and the recovered width parameters $\beta_{1}$ and $\beta_{2}$ of IDM agree well with the input ones, except near the maximum of the second wave train at $0.3 \mathrm{~Hz}$ (Fig. 2b). In this region, the intensity of the second wave train is smaller than $15 \%$ of the first and no information on it can be recovered. Figure $2 \mathrm{~d}$ shows that the mean directions of the wave trains recovered using IDM generally agree with those input to within $15 \%$.

In Fig. 2a, we show the spectrum $F(f)$ recovered from the elevation measurements along with a set of 

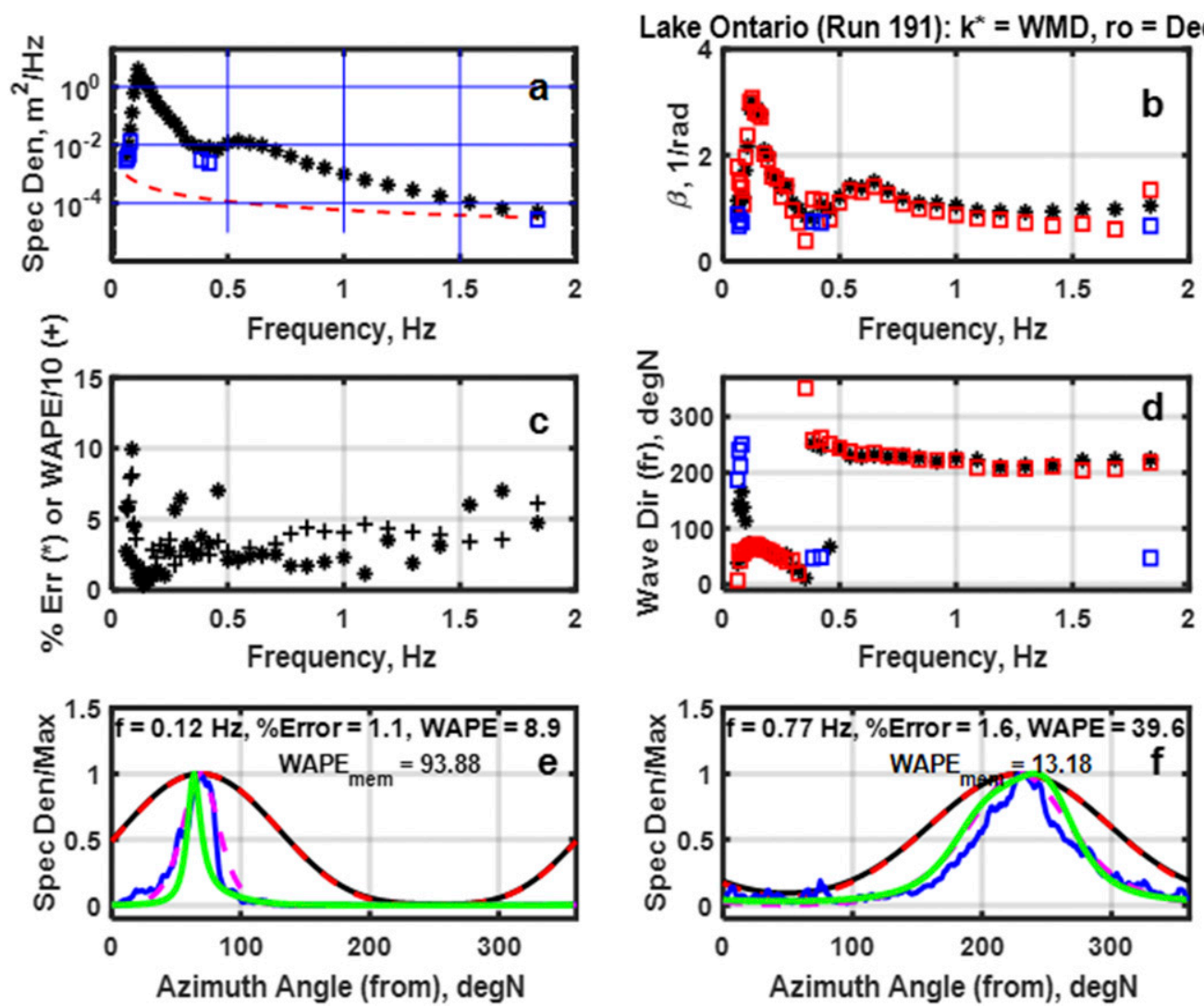

FIG. 5. As in Fig. 4, but for run 191, $\mathbf{U}_{10}=12.9 \mathrm{~m} \mathrm{~s}^{-1}$ from $220^{\circ} \mathrm{N}$.

smaller spectral values $F_{s}(f)$ recovered from the weaker, second peak of the spreading function using IDM. These have been recovered using the following equation:

$$
F_{s}(f)=\left[a \int_{-\pi}^{\pi} \Phi_{2}(\varphi) d \varphi / \int_{-\pi}^{\pi} \Phi(\varphi) d \varphi\right] F(f) .
$$

Here, $F_{s}(f)$ tracks the input $F_{2}(f)$ quite well.

In Fig. 2c, we show the weighted average percent error (WAPE) defined by Benoit (1994). This error measurement, which compares input and recovered spreading functions, is strongly affected by slight errors in the mean wave direction since spreading functions are fairly narrow. We prefer to measure the error as the percent error between the input and recovered $D$ since this determines the extent to which the measurements can be explained by the recovered spreading functions and it can be determined even when the true spreading function is unknown. These error functions are defined as follows:

$$
\mathrm{WAPE}=\frac{100 \sum \mid \Phi(\text { input })-\Phi(\text { recovered }) \mid}{\sum \Phi(\text { input })},
$$

$$
\% \text { Error }=\frac{\left.200 \sum \mid D \text { (input }\right)-D(\text { recovered }) \mid}{\left.\sum[D \text { (input })+D(\text { recovered })\right]} .
$$

Where possible, we will display both of these error measures in the figures below. In Fig. 2c, note the increase in the \%Error between frequencies of 0.27 and $0.33 \mathrm{~Hz}$, just before the second wave train begins to exceed $15 \%$ of the first. Since no $D$ (recovered) is given in the MEM method, we do not show a \%Error for MEM in Figs. 2e and 2f.

In Figs. 2e and 2f, we show the shapes of $D(f, \varphi)$ input and recovered by IDM and $\Phi(f, \varphi)$ input and recovered by IDM and MEM at two chosen frequencies, 0.12 and $0.77 \mathrm{~Hz}$. IDM recovers the input spreading function very closely at both frequencies. MEM does fairly well at $0.77 \mathrm{~Hz}$ but produces a bimodal spreading function at $0.12 \mathrm{~Hz}$. Note from Fig. 2a that at $0.12 \mathrm{~Hz}$, only the wave train generated by the $15 \mathrm{~m} \mathrm{~s}^{-1}$ wind exists; the bimodal spreading function recovered by MEM is incorrect.

Figure 3 shows the results for $\varphi_{1}=210^{\circ}, \varphi_{2}=120^{\circ}$, so the separation of the mean directions of the wave trains is $\Delta \varphi_{12}=\varphi_{1}-\varphi_{2}=90^{\circ}$. Since this separation is less than the width of the windowing function, IDM is unable to find two spreading functions when they exist. Instead, it 

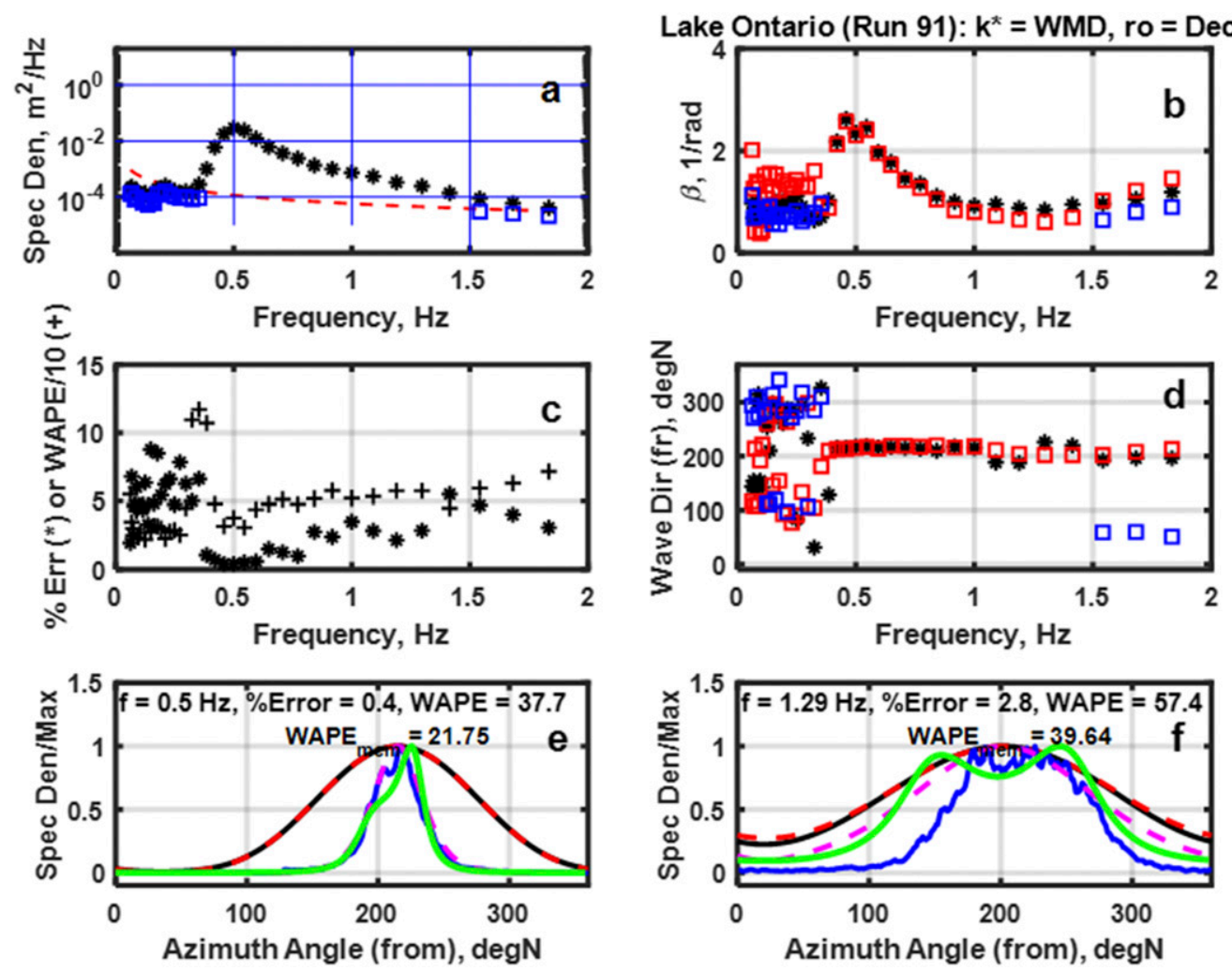

FIG. 6. As in Fig. 4, but for run $91, \mathbf{U}_{10}=11.4 \mathrm{~m} \mathrm{~s}^{-1}$ from $222^{\circ} \mathrm{N}$.

produces a broad spreading function whose mean direction is a weighted average of the two input directions and whose $\beta$ value lies below those of either wave train, indicating a broader spreading function. Both the WAPE and \%Error are increased where two wave trains exist. Where only one exists, IDM produces very small values of both error measures.

Figures $3 \mathrm{e}$ and $3 \mathrm{f}$ show that IDM does well at $0.12 \mathrm{~Hz}$ but errs in a known manner at $0.77 \mathrm{~Hz}$. MEM recovers fairly good spreading functions at $0.77 \mathrm{~Hz}$ but produces an incorrect bimodal spreading function at $0.12 \mathrm{~Hz}$.

\section{IDM and MEM recovered spreading functions compared with Lake Ontario WDM analysis}

In this section we utilize data collected on a tower owned by Canada's National Water Research Institute on Lake Ontario in 1987. The tower is located in the southwestern tip of Lake Ontario; a map of Lake Ontario with a blow-up of the area of the tower is given in Donelan et al. (1985). The long axis of the lake runs approximately along the direction $80^{\circ} \mathrm{N}$. Collected data consisted of wave height measurements made by a compact array of six capacitance wave probes arranged in a centered pentagon of radius $25 \mathrm{~cm}$. The data were processed to produce surface elevation along with north and east components of surface slope for waves up to $1.8 \mathrm{~Hz}$ in frequency at a sample rate of $4 \mathrm{~Hz}$. These height-slope data were averaged into 40 logarithmically space frequency bins and processed as outlined in section 1. IDM and MEM were then used to recover estimates of the directional wave spectra using the five Fourier components from standard processing techniques. The data were also processed using the WDM to produce directional wave spectra. This technique using Morlet wavelets has been described in detail elsewhere (Donelan et al. 1996, 2015). WDM differs from other methods in that the wavenumber and amplitude of each wave in each frequency band are identified at each sampling time step. WDM derives direction from phases of the wavelet transforms of the surface elevation data at three or more wave staffs. WDM never uses the five Fourier coefficients used in standard processing. Spectra recovered using these very different methods are compared below.

We show the results of these comparisons in Figs. 4-6. Three runs from the 1987 Lake Ontario data were used: runs 189, 191, and 91 with wind velocities at $10 \mathrm{~m}$, $\mathbf{U}_{10}=11.4 \mathrm{~m} \mathrm{~s}^{-1}$ from $84^{\circ} \mathrm{N}, 12.9 \mathrm{~m} \mathrm{~s}^{-1}$ from $220^{\circ} \mathrm{N}$, and $11.3 \mathrm{~m} \mathrm{~s}^{-1}$ from $222^{\circ} \mathrm{N}$, respectively. The IDM results all show evidence of both weak and strong 


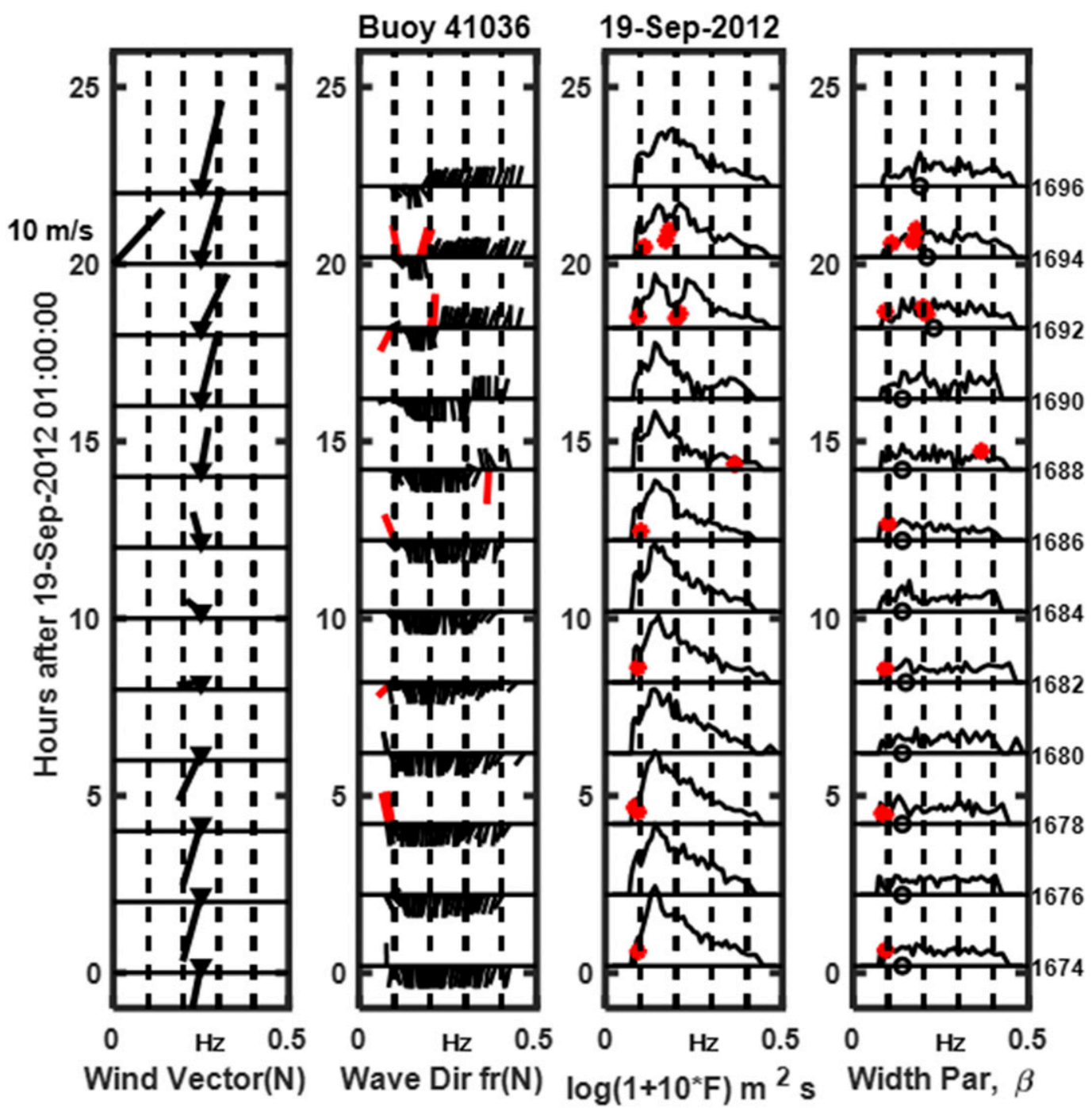

FIG. 7. (left to right) Wind vectors, wave directions (from: toward zero), omnidirectional wave spectra, and width parameter $\beta_{1}$. The last three parameters are plotted vs wave frequency. The data in panels 2,3 , and 4 , along with the assumed $\mathrm{sech}^{2}$ form for the spreading function, completely specify the directional wave spectrum. Horizontal lines in panel 3 indicate values of $\log [1+10 F(f)]$ of -1 and $25 \mathrm{in} \mathrm{m}^{2} \mathrm{~Hz}^{-1}$ for the wave spectrum at that time step. Horizontal lines in panel 4 indicates values of $\beta_{1}$ of 0 and 6 . Red symbols in panels 2-4 indicate secondary wave trains. Open circles in panel 4 show the dominant wave frequency. Numbers to the left of each panel indicate the number of hours after midnight on that day. Numbers to the right of panel 4 give the number of the spectrum starting at the beginning of the run.

peaks at some frequencies. Not all of the weak peak results are real due to noise considerations. Figure $6 \mathrm{a}$ shows that the noise floor at a frequency of $0.2 \mathrm{~Hz}$ is about $3 \times 10^{-4} \mathrm{~m}^{2} \mathrm{~Hz}^{-1}$. Because our frequency bins are logarithmically spaced, however, this noise floor varies according to $N=3 \times 10^{-4} d f(0.2) / d f$. This noise floor is shown in Figs. 4a, 5a, and 6a as a red dashed curve. If the measured spectral density is near the noise floor, then the measured heights and slopes will be contaminated by noise and could yield incorrect peaks in the measured spreading function. Thus, we will ignore the weaker of two peaks at frequencies where spectral densities are near the noise floor. We will show, however, that not all weaker spreading functions at a given frequency are noise related.

In Fig. 4, for run 189, both WDM and IDM show that a wave train with a very narrow spreading function is propagating from $80^{\circ} \mathrm{N}$, along the axis of the lake, for frequencies below $0.4 \mathrm{~Hz}$ while a broader wave train from $110^{\circ} \mathrm{N}$ begins to be visible at a frequency of about $0.4 \mathrm{~Hz}$. The agreement is very good between results produced by the two methods for mean wave 

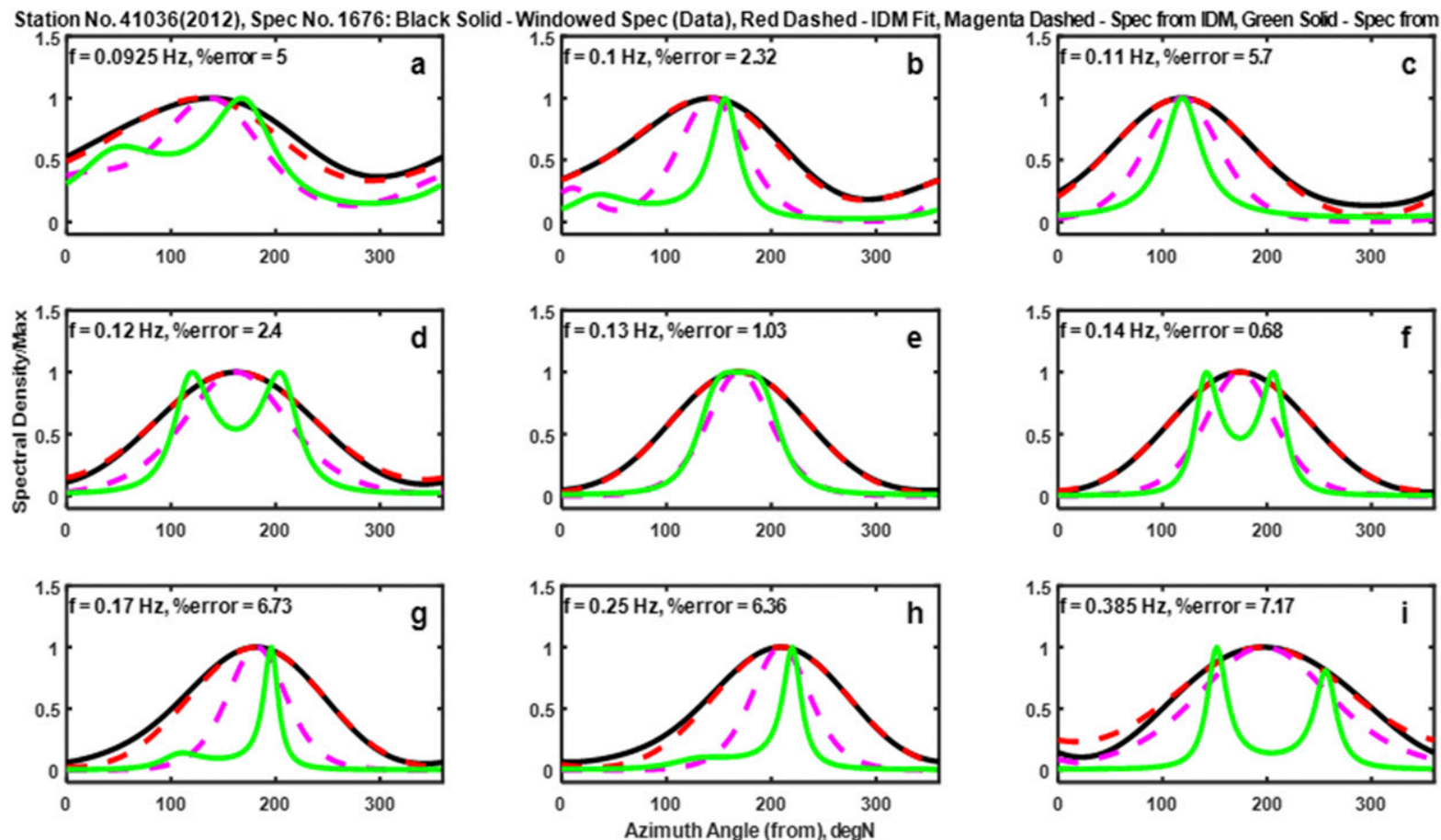

FIG. 8. Plots at selected frequencies of the spreading function measured by the buoy $D(f, \varphi)$ and the true spreading function $\Phi(f, \varphi)$. Solid black curves are the buoy-measured spreading function, dashed red curves are the IDM-recovered $D(f, \varphi)$, dashed magenta curves are the IDM-recovered $\Phi(f, \varphi)$, and green curves are the MEM-recovered $\Phi(f, \varphi)$. The spreading functions all come from spectrum 1676 shown in Fig. 7, which is before the wind shift.

directions and the width parameter $\beta_{1}$. The data approach the noise floor for frequencies below about $0.1 \mathrm{~Hz}$ and above $1.75 \mathrm{~Hz}$ so neither method gives reliable results at these low and high frequencies. In the midrange of frequencies, IDM shows weak second peaks at many frequencies. These appear to be real although they did not appear in the WDM results since it is unable to separate two wave trains at the same frequency. This secondary wave train propagates from approximately north. The fetch in this direction is quite short so the narrow spreading functions (high $\beta$ values) are expected. Spreading function shapes recovered by IDM and MEM agree very well with WDM at $0.12 \mathrm{~Hz}$. At $0.77 \mathrm{~Hz}$, IDM and MEM yield comparable fits to the WDM spreading function but neither fits well.

In Fig. 5, for run 191, both WDM and IDM show that a narrow wave train is also present in this run up to frequencies of about $0.4 \mathrm{~Hz}$ with waves coming from directions that rotate from $80^{\circ}$ to $40^{\circ} \mathrm{N}$ with increasing frequency. At $0.4 \mathrm{~Hz}$, a second wave train becomes visible with intensity similar to that in Fig. 4 but more visible due to a decrease in the intensity of waves just below $0.4 \mathrm{~Hz}$. Both WDM and IDM show that this wave train comes from about $210^{\circ} \mathrm{N}$, a short fetch direction. In all cases, $\beta$ values and mean wave directions from WDM and IDM processing agree very well. Two viable wave trains at a given frequency are recovered by the IDM analysis only at frequencies around $0.4 \mathrm{~Hz}$ where the two wave trains are of comparable amplitude; the other cases are noise related. In all cases, \% Error is very low where the wave intensity is large and never exceeds $10 \%$. WAPE, on the other hand, is much larger than this since, as mentioned, a small difference in wave direction causes a large increase in WAPE. Spreading function shapes recovered by IDM and MEM agree well with those from WDM at both 0.12 and $0.77 \mathrm{~Hz}$, although the MEM spreading function at $0.12 \mathrm{~Hz}$ appears to be too narrow.

Figure 6 shows results for Lake Ontario data of run 91 during 1987. Here again, we observe a wave train coming from about $210^{\circ} \mathrm{N}$ and peaking at $0.5 \mathrm{~Hz}$, close to the values observed in Fig. 5. This wind direction is offshore and the fetch is about $1 \mathrm{~km}$, accounting for the small observed spectral densities. Below $0.4 \mathrm{~Hz}$, the data are noise as the erratic behavior of the wave directions indicates. Above that frequency, however, the data processed with WDM and IDM agree very well and show the expected behavior of $\beta$, peaking at about 2.5 at the dominant wave frequency and falling to lower values above that. At frequencies higher than $0.4 \mathrm{~Hz}, \%$ Error is below about $6 \%$ but WAPE is quite high. All examples of double-peaked spreading functions 

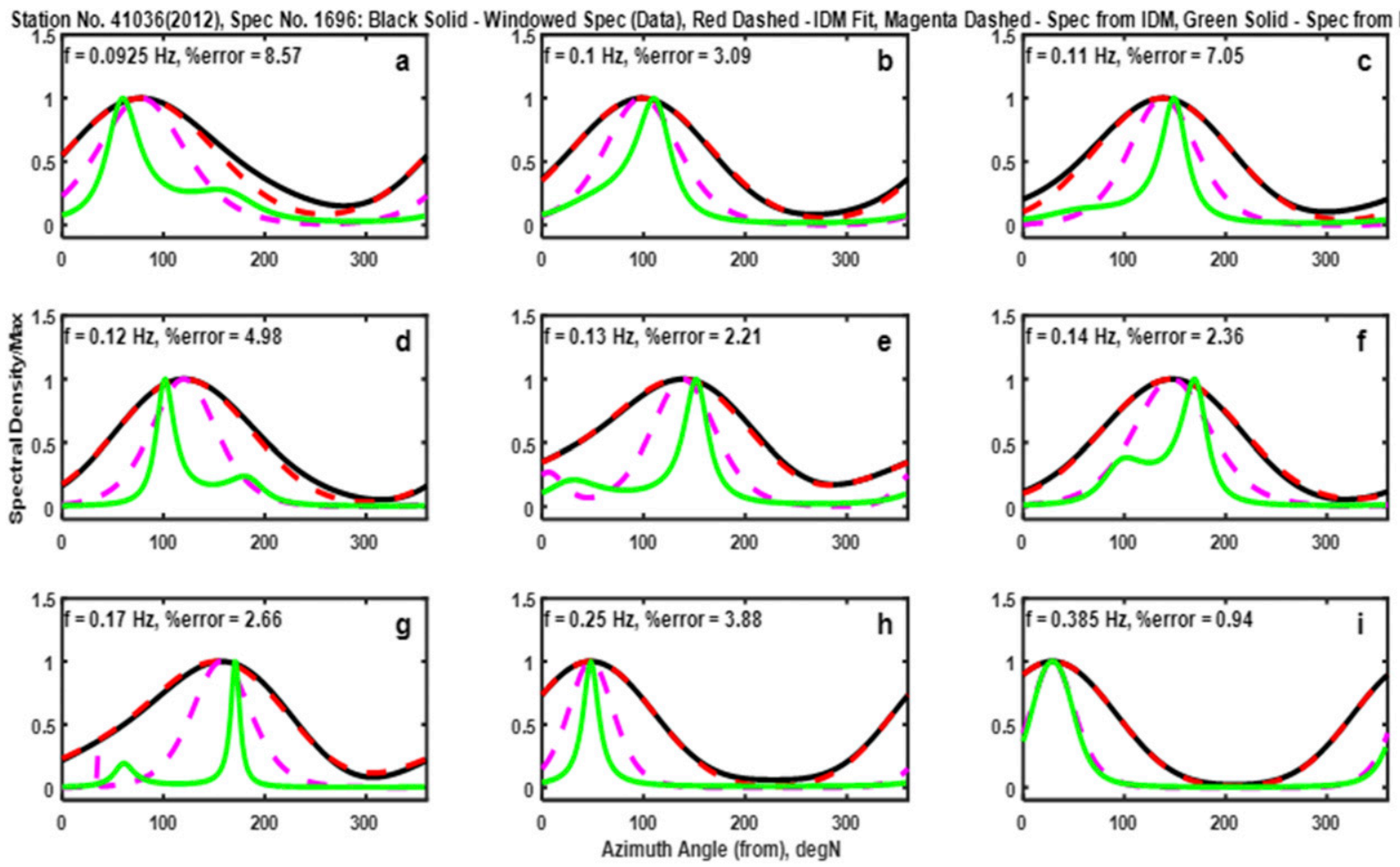

FIG. 9. As in Fig. 8, but for spectrum 1696, which is after the wind shift.

at a single frequency recovered by IDM appear to be noiseinduced. At $0.12 \mathrm{~Hz}$, spreading functions from both IDM and MEM agree with the one obtained by WDM very well. At $0.77 \mathrm{~Hz}$, however, both appear to be too broad and the one from MEM is questionably double peaked.

\section{Examples of IDM and MEM recoveries from the open ocean}

We applied IDM to open ocean directional spectra obtained from the NDBC website (ndbc.noaa.gov/data/ historical/). At this point in time, we have processed nearly 185000 directional spectra to correct their widths. A convenient way to display time series of these processed data is shown in Fig. 7. Data from buoy 41036, which is off the North Carolina coast, are displayed every $2 \mathrm{~h}$ for 1 day with earlier times at the bottom. Counting panels from the left, the first panel shows the direction from which the wind comes as the direction toward which the needle points (like an airport wind sock). Wind speed is indicated by the length of the needle (or vector). The second panel shows the wave directions at the 47 frequencies given by NDBC. The format of the vectors is the same as in the first panel except that vector lengths are constant. The third panel shows the omnidirectional wave spectrum of the primary waves in black. Finally, the irregular black lines in the fourth panel shows the width parameter $\beta_{1}$ at each frequency. Because IDM assumes a parametric form for the spreading function, the data in Fig. 7 are all that are required to specify the directional wave spectrum every $2 \mathrm{~h}$.

The wind over this 24 -h period rotated clockwise and the shorter waves followed shortly after, longer ones changing later. Figures 8 and 9 show a selection of plots of $D(f, \varphi)$, as measured by the buoy and recovered by IDM, and $\Phi(f, \varphi)$ as recovered by IDM and MEM. Clearly, the shapes of $\Phi(f, \varphi)$ as given by MEM are not reliable in either their width or direction. The turning of the waves to follow the wind would be difficult to see if only MEM were available.

Figure 10 shows wind and wave spectral parameters every $2 \mathrm{~h}$ for buoy 46087 located at the mouth of the Strait of Juan de Fuca. The strait is about $15 \mathrm{~km}$ wide for the $80 \mathrm{~km}$ leading to the mouth. The axis of the strait here is along a direction of $110^{\circ} \mathrm{N}$, which is also the direction from which the wind comes on the day illustrated in Fig. 10. Therefore, a high wind of 12$15 \mathrm{~m} \mathrm{~s}^{-1}$ is blowing to the west directly along the axis of the strait on this January day. Figure 10 shows that this wind blowing down through the narrow strait produces a very narrow surface wave spreading function. Values of $\beta_{1}$ reach as high as 4 or 5 (recall that $\beta_{1}$ is usually around 2 at the spectral peak and that larger values of $\beta_{1}$ indicate narrower spreading functions). Notice that waves at 


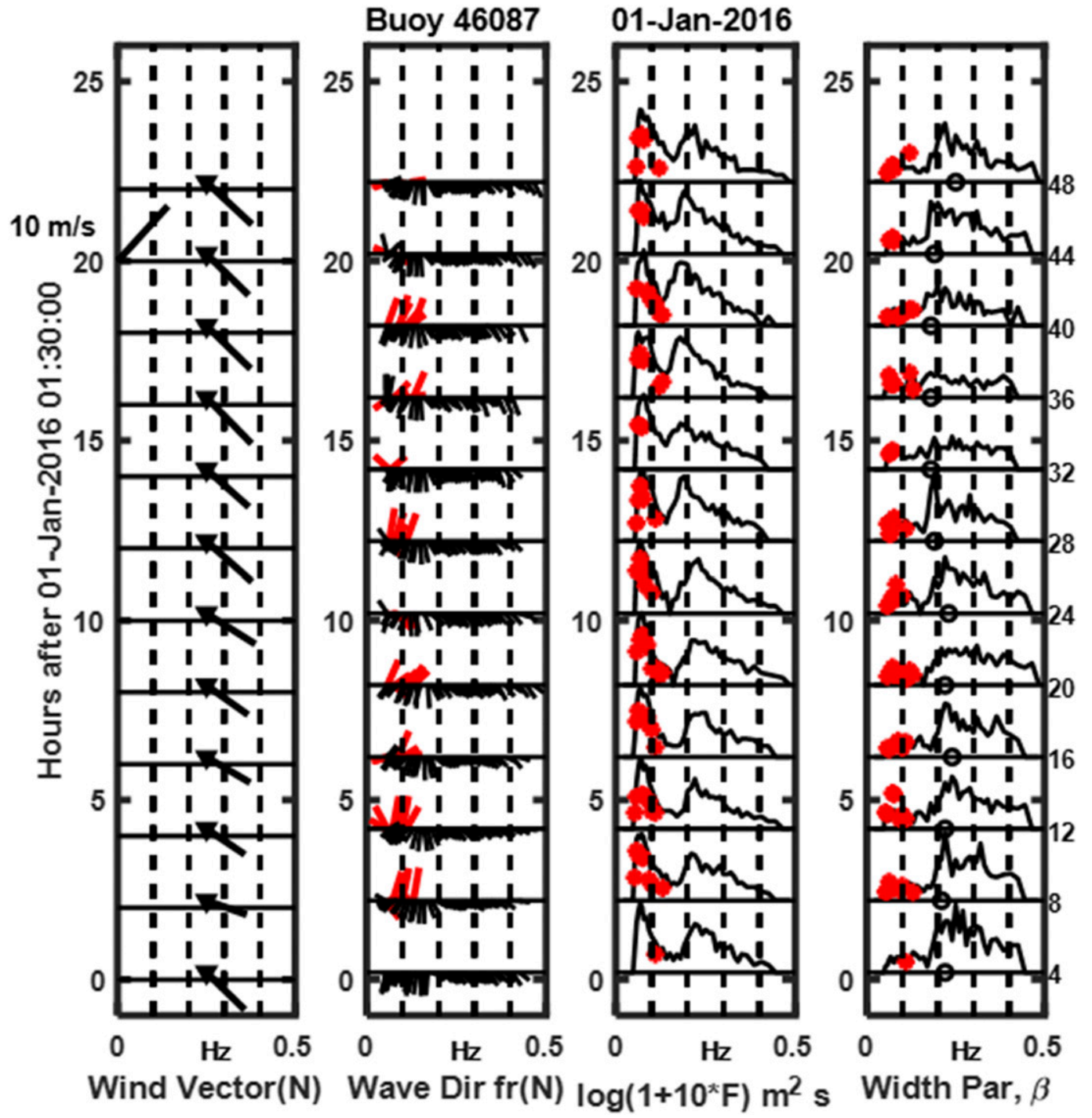

FIG. 10. As in Fig. 7, but near the mouth of the Strait of Juan de Fuca.

frequencies below about $0.2 \mathrm{~Hz}$ do not align with the wind in Fig. 10 and that the peak frequency lies close to the lowest frequency that does align with the wind. Furthermore, the peak frequency is near $0.2 \mathrm{~Hz}$ over the whole 24-h period, indicating that the wind waves here are fully developed. The red asterisks and vectors and the black vectors below $0.2 \mathrm{~Hz}$ indicate that swell from the ocean is arriving at this buoy.

Figure 11 shows plots of the spreading function measured by buoy $46087 D(f, \varphi)$ and the true spreading function $\Phi(f, \varphi)$ at various selected frequencies for spectrum 33 of Fig. 10. As in Figs. 8 and 9, MEM often produces bimodal spreading functions, which are implausible for a spectrum obtained in a narrow channel with a high wind speed along its axis. It is more plausible that, as IDM indicates, the wave spectrum narrows under these circumstances due to bottom friction and reflections acting on waves at large angles to the wind.

\section{Conclusions}

We have described a new method for recovering directional ocean wave spectra from surface height and slope measurements taken over a small area, the iterative deconvolution method (IDM), and compared it with a more standard method, the maximum entropy method. We have tested both of these methods against simulated data and found that IDM does a very good job of retrieving the input directional spectra if a single wave train is present. It also works well in the case of two wave trains if the angle between the trains 

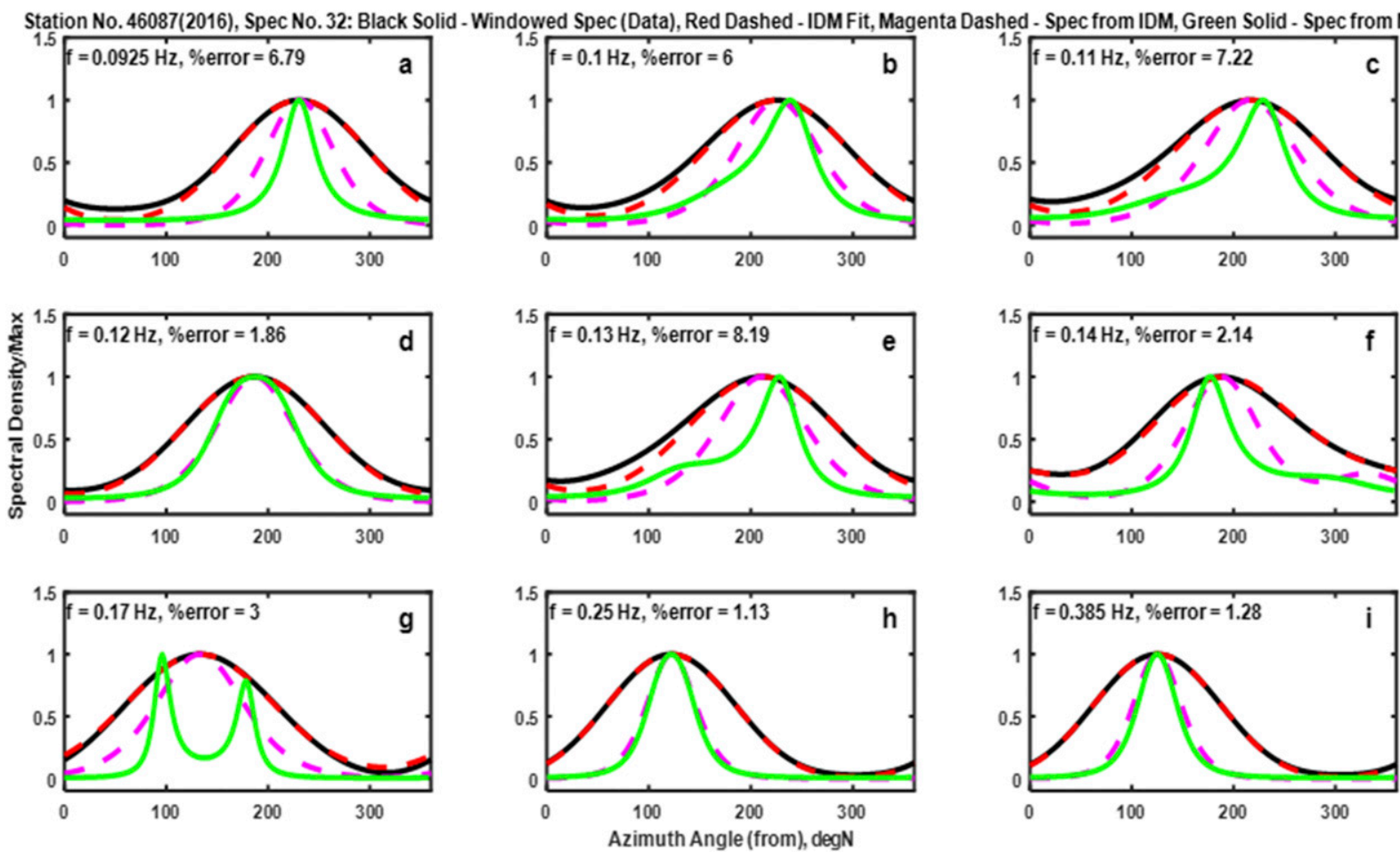

FIG. 11. As in Fig. 8, but for buoy 46087 in 2016. This corresponds to spectrum 32 in Fig. 10.

approaches or exceeds the width of the windowing function. Thus, IDM can address the question of "bimodal spectra" if the two peaks are separated by more than some minimum angle on the order of $130^{\circ}$, which often seems to be the case (Hwang et al. 2015). Maximum entropy method (MEM), on the other hand, seems to work better if two wave trains are present than if only one exists. For two input wave trains, MEM produces spreading functions that show two peaks near the proper frequency, but they are narrower than the input spectral peaks. In the single-wave-train case, MEM, as reported by Benoit (1994), often produces incorrect bimodal spectra. We have also compared both methods to the wavelet directional method (WDM) for computing directional spectra. We find that IDM and MEM give very similar results to those produced by WDM, but that MEM occasionally produces spectra that are narrower than those given by the other two methods. IDM recovers weak spreading functions that MEM and WDM do not but the stronger spreading functions from all methods agree well in mean direction and width.

Finally, we applied IDM and MEM to two datasets collected by the National Data Buoy Center's directional wave buoys, which are available on the NDBC website. First, we looked at two days on which 24 and 48 spectra were collected by NDBC every hour or half hour. In one case, data were collected off the east coast of the United States during a period when the wind shifted rapidly from about $240^{\circ} \mathrm{N}$ to come from about $40^{\circ} \mathrm{N}$. Spreading functions obtained with IDM clearly showed the higher frequency waves following this wind shift with lower-frequency waves following progressively later. MEM again frequently produced bimodal spreading functions in which the two peaks were separated by large angles, making it difficult to find evidence of the short waves following the wind.

The second dataset we investigated was a series of directional spectra taken every half hour by an NDBC buoy near the mouth of the Strait of Juan de Fuca. Here the wind was high and directed from east to west along the axis of the strait where it is $15 \mathrm{~km}$ wide. The IDM analysis clearly showed that mean directions of the shorter waves were along the axis of the strait and that the spreading functions of these waves were unusually narrow, probably due to bottom friction acting on waves traveling at large angles to the wind. Furthermore, the analysis showed that waves below about $0.2 \mathrm{~Hz}$ did not travel primarily in the wind direction and that the spectrum of waves that were in the wind direction became fully developed at nearly the frequency where the waves stopped traveling in the wind direction. MEM, as usual, often produced bimodal spectra but the two peaks did not appear to be narrower than those it produced in the East Coast study. The narrowing of the spreading function showed by IDM was difficult to detect in the MEM analysis. 
In conclusion, the IDM was shown by this study to produce true spreading functions that agreed with simulated input and with spectra measured with the WDM. It was also able to detect short waves turning in a turning wind and their spreading function narrowing upon propagating through a narrow strait. The MEM seemed to be a much less reliable method of attempting to determine the spreading function. Its tendency to produce bimodal spectra with widely separated peaks made it less than optimum to do directional wave studies. Even when it did not produce double peaks, it produced spreading functions that were generally too narrow. Due to the lack of alternative methods of obtaining true directional wave spectra, MEM had never been validated to our knowledge prior to this study, except for the studies of Benoit (1994) using simulated data and Donelan et al. (2015) using field data and WDM. In the study of Donelan et al. both MEM and the maximum likelihood method (MLM) were shown to sometimes produce incorrect bimodal spectra. We tried varying the input parameters to the MEM function but could find no combination of slightly different parameters that produced better results. We believe that the good agreement of IDM output with the directional spectra obtained from the nonstandard WDM validates both methods. We recommend their use.

Acknowledgments. Mark Donelan died midway through the research leading to this paper. Without his input, this would have been a very different paper. Mark suggested the comparisons with WDM, did the WDM analysis, and, long ago, collected the Lake Ontario data. He felt strongly about the need for simulations to see whether IDM could separate two spreading functions at the same frequency. I very much miss his counsel on air-sea interaction issues as well as his gentle encouragement on other issues. This research was sponsored by Eric Lindstrom through NASA Grant NNX17AH23G Programs and data used in this paper are available on figshare (10.6084/m9.figshare.6987323). Search for William Plant. The iterative deconvolution method is implemented in the MATLAB function "IDM_Directional_Estimator.m." This function can also be obtained by emailing plant@apl.washington.edu.

\section{APPENDIX A}

\section{Nonunique Weighting Functions}

The measured time series are height $\gamma(t)$, north slope $s_{n}(t)$, and east slope $s_{e}(t)$, whose complex Fourier transforms (FFTs) are $\tilde{\gamma}(f)=\int_{-\pi}^{\pi} \tilde{\gamma}\left(f, \varphi^{\prime}\right) d \varphi^{\prime}, \tilde{s}_{n}=\int_{-\pi}^{\pi} k \cos \varphi^{\prime} \tilde{\gamma}\left(f, \varphi^{\prime}\right) d \varphi^{\prime}$, and $\tilde{s}_{e}=\int_{-\pi}^{\pi} k \sin \varphi^{\prime} \tilde{\gamma}\left(f, \varphi^{\prime}\right) d \varphi^{\prime}$. If we define the nondirectional wave spectrum $F(f)$ to be $\left\langle|\tilde{\gamma}|^{2}\right\rangle / \Delta f$, then Eq. (2) can be written

$$
\begin{aligned}
& a_{o}=\frac{F(f)}{\pi}=\frac{1}{\pi} \int_{-\pi}^{\pi} F\left(f, \varphi^{\prime}\right) d \varphi^{\prime}, \\
& a_{1}=-\frac{1}{\pi} \int_{-\pi}^{\pi} F\left(f, \varphi^{\prime}\right) \sin \varphi^{\prime} d \varphi^{\prime}, \\
& b_{1}=-\frac{1}{\pi} \int_{-\pi}^{\pi} F\left(f, \varphi^{\prime}\right) \cos \varphi^{\prime} d \varphi^{\prime}, \\
& a_{2}=\frac{1}{\pi} \int_{-\pi}^{\pi} F\left(f, \varphi^{\prime}\right)\left(\cos ^{2} \varphi^{\prime}-\sin ^{2} \varphi^{\prime}\right) d \varphi^{\prime}, \\
& b_{2}=\frac{2}{\pi} \int_{-\pi}^{\pi} F\left(f, \varphi^{\prime}\right) \cos \varphi^{\prime} \sin \varphi^{\prime} d \varphi^{\prime} .
\end{aligned}
$$

We may write a generalization of Eq. (3), in which the coefficients of the terms are undetermined, as

$$
\begin{aligned}
F_{B}(f, \varphi)= & \frac{1}{2 \pi} \int_{-\pi}^{\pi} F\left(f, \varphi^{\prime}\right) d \varphi^{\prime}+\frac{A_{1}}{\pi} \int_{-\pi}^{\pi} F\left(f, \varphi^{\prime}\right)\left[\sin \varphi^{\prime} \sin \varphi+\cos \varphi^{\prime} \cos \varphi\right] d \varphi^{\prime} \\
& +\frac{B_{1}}{\pi} \int_{-\pi}^{\pi} F\left(f, \varphi^{\prime}\right)\left[\cos ^{2} \varphi^{\prime}-\sin ^{2} \varphi^{\prime} \cos 2 \varphi+2 \cos \varphi^{\prime} \sin \varphi^{\prime} \sin 2 \varphi\right] d \varphi^{\prime},
\end{aligned}
$$

which may be written,

$$
\begin{aligned}
F_{B}(f, \varphi)= & \frac{1}{2 \pi} \int_{-\pi}^{\pi} F\left(f, \varphi^{\prime}\right) d \varphi^{\prime} \\
& +\frac{A_{1}}{\pi} \int_{-\pi}^{\pi} F\left(f, \varphi^{\prime}\right) \cos \left(\varphi^{\prime}-\varphi\right) d \varphi^{\prime} \\
& +\frac{B_{1}}{\pi} \int_{-\pi}^{\pi} F\left(f, \varphi^{\prime}\right) \cos \left[2\left(\varphi^{\prime}-\varphi\right)\right] d \varphi^{\prime},
\end{aligned}
$$

or, finally,

$$
F_{B}(f, \varphi)=\frac{1}{2 \pi} \int_{-\pi}^{\pi} F\left(f, \varphi^{\prime}\right) W\left(\varphi^{\prime}-\varphi\right) d \varphi^{\prime},
$$

where

$$
W\left(\varphi^{\prime}-\varphi\right)=1+2 A_{1} \cos \left(\varphi-\varphi^{\prime}\right)+2 B_{1} \cos 2\left(\varphi-\varphi^{\prime}\right) .
$$



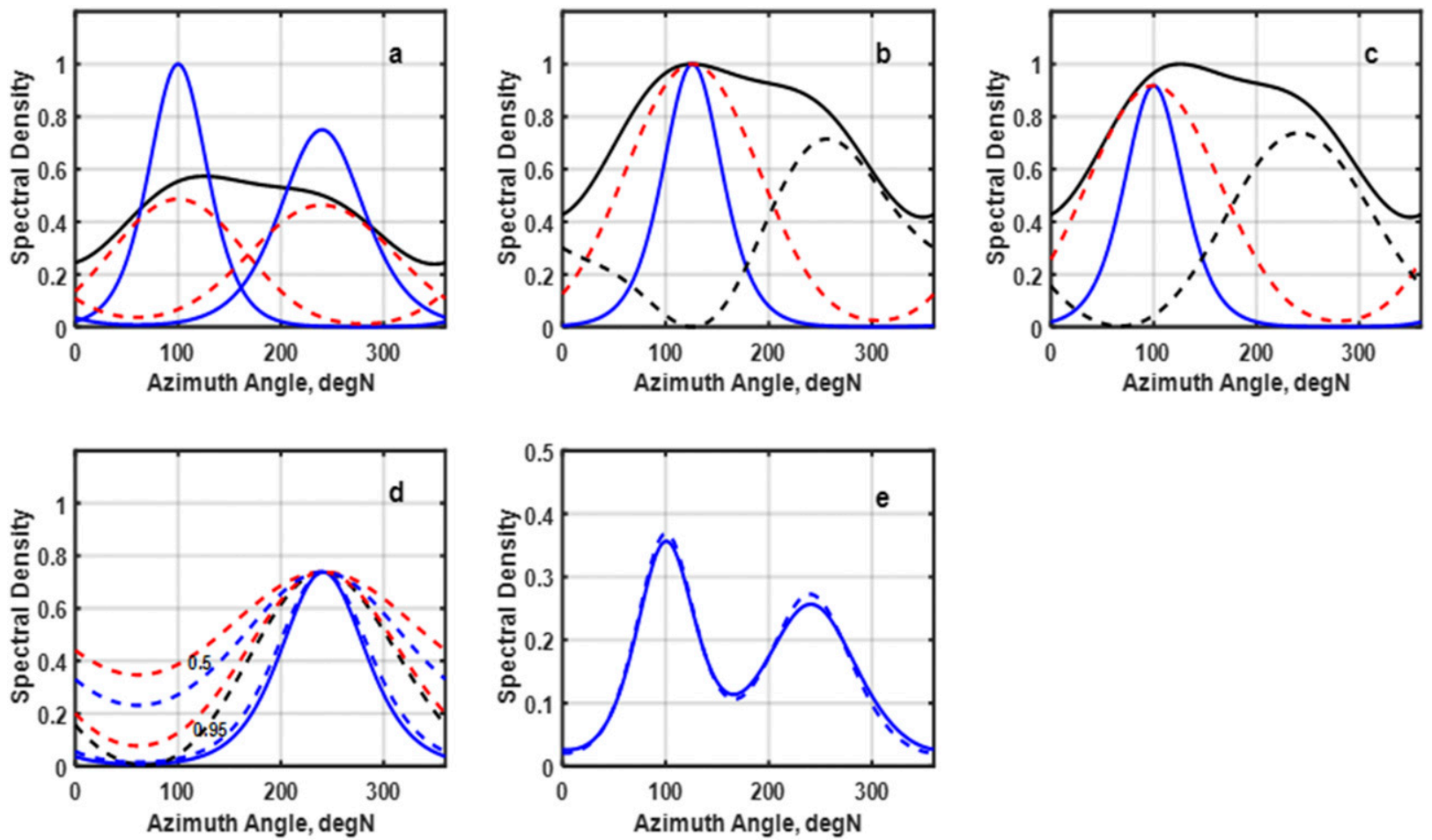

FIG. B1. Iterative deconvolution for a spreading function with two peaks. (a) The blue curves are the two spreading functions, $\Phi_{1}$ maximizing at 1 and $a \Phi_{2}$ maximizing at 0.75 , the red dashed curves are their convolutions with $W$ (normalized to integrate to 1 ), and the black curve is the sum of the red curves $D$. (b) The peak of $\left(\Phi_{1}{ }^{*} W\right) / \max \left(\Phi_{1}{ }^{*} W\right)$ is shifted to the maximum of $D / \max (D)$ and subtracted from $D / \max (D)$ to yield $f_{r}$ (black dashed curve). (c) The peak of $\Phi_{1} / \max \left(\Phi_{1}\right)$ is again shifted by an amount determined from Fig. B2. Subtracting from $D / \max (D)$ now yields $f_{r n}$ (black dashed curve). The height of $\Phi_{1}$ is iteratively reduced by $c_{r}$ until $f_{r n}$ is positive everywhere. (d) $\beta_{2}$ is now iteratively increased as $\beta_{1}$ was in Fig. 1 until $f_{r n}$ is fit. (e) $\Phi_{1}$ and $a \Phi_{2}$ are now added to yield the recovered spreading function (here normalized so that it integrates to 1 ) (solid blue curve). The dashed curve is the normalized sum of the two solid blue curves in (a), which was our initial spreading function.

If $A_{1}=2 / 3$ and $B_{1}=1 / 6$, Eq. (A5) yields the LCS result, Eq. (5), but note that this result for $W$ is not unique and therefore the form of $F_{B}(f, \varphi)$, or more precisely, $D(f, \varphi)$ of Eq. (1) is not unique. This does not change the fact that $F(f, \varphi)$ is unique. The iterative deconvolution method can be used no matter what form is chosen for $W$. However, varying the coefficients $A_{1}$ and $B_{1}$ indicates that suitable windowing functions are difficult to produce. Narrow windows generally have more than a single maximum.

\section{APPENDIX B}

\section{Recovering Two Wave Trains}

Here we consider the case where two wave trains exist at a single frequency. The process becomes considerably more complicated than if only a single wave train exists. In this case, $\Phi=\Phi_{1}+a \Phi_{2}$, where $a(\leq 1)$ determines the relative amplitudes of the two spreading functions and $\Phi_{2}=\operatorname{sech}^{2}\left\{\beta_{2}(f)\left[\left(\varphi-\varphi_{2}(f)\right]\right\}\right.$. Then we have

$$
D(f, \varphi)=\Phi_{1}\left(f, \varphi-\varphi_{1}\right) * W(\varphi)+a \Phi_{2}\left(f, \varphi-\varphi_{2}\right) * W(\varphi),
$$

as shown in Fig. B1a. Initially we know neither $\varphi_{1}$ nor $\varphi_{2}$ so the best we can do is set $\varphi_{1}=\varphi_{D}$ and $\varphi_{2}=\varphi_{r}$, where $\varphi_{r}$ is unknown. Then, as with a single wave train, we consider the difference between the measured buoy spectrum $D$ and the convolution of the windowing function and an initial $\Phi_{1}$ having a small value of $\beta_{1}$ :

$$
\frac{D(f, \varphi)}{\max (D)}-\frac{\Phi_{1}\left(f, \varphi-\varphi_{D}\right) * W(\varphi)}{\max \left(\Phi_{1} * W\right)}=f_{r}\left(f, \varphi-\varphi_{r}\right)
$$

We then iteratively increase $\beta_{1}$ until $f_{r}$ first becomes greater than -0.001 everywhere. This is where we would stop if only one wave train existed on the surface but, as Fig. B1b shows, if a second significant train exists on the surface, the resulting residual function $f_{r}$ may show a definite maximum at some value of $\varphi$. If this maximum exceeds 0.15 , we conclude that a second wave 


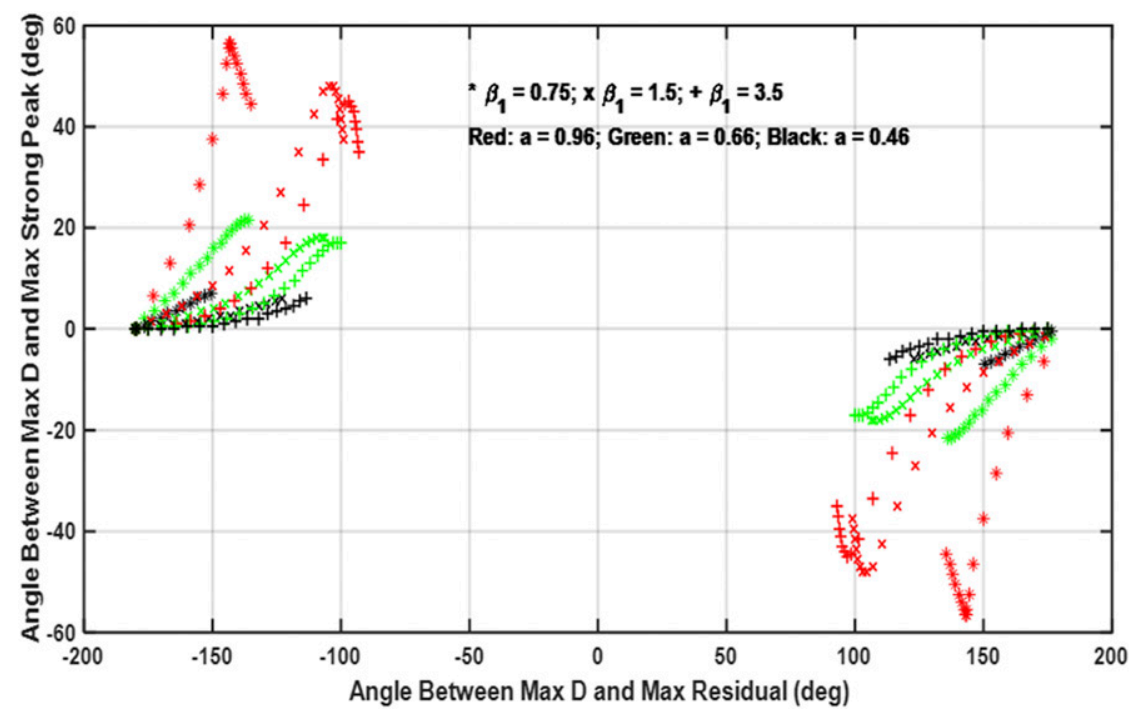

FIG. B2. The dependence of the shift of the angle between the peak of $\Phi_{1}$ and the peak of $D\left(\Delta \Phi_{1 D}\right)$ on the angle between the peak of the residual and the peak of $D\left(\Delta \Phi_{r D}\right)$ for various $\beta_{1}$ values and various amplitudes of the weaker peak $a$.

train exists and take this value of $\varphi$ to be $\varphi_{r}$ and the value of $f_{r}$ at $\varphi_{r}$ to be $a$. We continue increasing $\beta_{1}$ until $\varepsilon_{2}$ in the following equation is first greater than or equal to -0.001 :

$$
\begin{gathered}
\left.\frac{D(f, \varphi)}{\max (D)}\right|_{1 / 2}-\left.\frac{\Phi_{1}\left(f, \varphi-\varphi_{D}\right)^{*} W(\varphi)}{\max \left(\Phi_{1}{ }^{*} W\right)}\right|_{1 / 2} \\
-\left.\frac{a \Phi_{2}\left(f, \varphi-\varphi_{r}\right)^{*} W(\varphi)}{\max \left(\Phi_{2}{ }^{*} W\right)}\right|_{1 / 2} \\
=\varepsilon_{1}-\left.\frac{a \Phi_{2}\left(f, \varphi-\varphi_{r}\right)^{*} W(\varphi)}{\max \left(\Phi_{2}{ }^{*} W\right)}\right|_{1 / 2}=\varepsilon_{2} .
\end{gathered}
$$

Note that $\varepsilon_{1}=\left.f_{r}\left(f, \varphi-\varphi_{r}\right)\right|_{1 / 2}$, the value of $f_{r}$ evaluated at the angle where $D$ is at half power on the side falling most rapidly. This procedure causes $\Phi_{1}$ to be narrower than it would be if $a=0$, that is, if the spreading function were single peaked.

Two problems now occur. One is that, while we vary $\beta_{1}$ in $\Phi_{1}$ we do not know what value to use for $\beta_{2}$ in $\Phi_{2}$. We find that we obtain the most accurate $\beta_{1}$ if we let $\beta_{2}=0.7 \beta_{1}$ at this step. Second, if two wave trains exist at a single frequency, then in general $D$ is not symmetric in $\varphi$ and its maximum does not occur at the maximum of either $\Phi_{1}$ or $\Phi_{2}$ (see Fig. B1b). Setting the angle of the maximum of $\Phi_{1}$ to that of $D$ therefore does not yield the correct function for either $\Phi_{1}$ or $\Phi_{2}$. We make the assumption, though, that the value of $\beta_{1}$ we recover is the correct value. Therefore, we only need to shift the maximum of $\Phi_{1}$ to its proper location. We determine the necessary shift by simulating $f_{r}$. To do this, we use known $\operatorname{sech}^{2}$ functions for $\Phi_{1}\left(\varphi-\varphi_{1}\right)$ and $\Phi_{2}\left(\varphi-\varphi_{2}\right)$, and obtain $D$ by convolving them with $W$ and adding (see Fig. B1a). We do this for various values of $a, \beta_{1}$, and $\varphi_{2}$ for a given $\varphi_{1}$. Subtracting $\Phi_{1} * W / \max \left(\Phi_{1} * W\right)$ evaluated at $\varphi_{D}$ from $D / \max (D)$ as in Eq. (B2) then yields $f_{r}$ (see Fig. B1c) so we can determine the angular separation of the maxima of $f_{r}$ and $D, \Delta \varphi_{r D}=\varphi_{r}-\varphi_{D}$. It is then straightforward to determine how the shift of the maximum of $\Phi_{1}$ from the maximum of $D, \Delta \varphi_{1 D}=\varphi_{r}-\varphi_{D}$, depends on $\Delta \varphi_{r D}$ for various values of $a, \beta_{1}$, and $\varphi_{2}$ for a given $\varphi_{1}$ (see Fig. B2).

Having determined these dependences, we can now shift the peak of $\Phi_{1}$ accordingly since the data yield $a, \beta_{1}$, and $\Delta \varphi_{r D}$. But, as Fig. B1c shows, the amplitude of $\Phi_{1} * W$ must be reduced to match $D / \max (D)$ at its new angular location in order to keep the new residual function $f_{r n}$ positive. Then, recalling that $\varepsilon_{2}$ in Eq. (B3) is negligibly small, we have

$$
f_{r n}(\varphi)=\frac{D(\varphi)}{\max (D)}-\frac{c_{r} \Phi_{1}\left(\varphi-\varphi_{1}\right)^{*} W}{\max \left(\Phi_{1}{ }^{*} W\right)}=\frac{c_{r} a \Phi_{2} * W}{\max \left(\Phi_{2} * W\right)}
$$

Continuously reducing the value of $c_{r}(\leq 1)$ until first $f_{r n}(\varphi)>-0.001$ for all $\varphi$ yields $c_{r} a \Phi_{2} * W / \max \left(\Phi_{2}{ }^{*} W\right)$ for that value of $\beta_{2}$. On the rare occasions where the width of $f_{r n}$ is less than the width of $W$, we cannot recover a value for $\beta_{2}$. In the vast majority of cases, though, $f_{r n}$ is wider than $W$ and we use the same procedure as for a single spreading function. We begin iterating the function $c_{r} a \operatorname{sech}^{2}\left[\beta_{2}\left(\varphi-\varphi_{r}\right)\right]$ starting with small values of $\beta_{2}$ and iteratively increasing it until we match $c_{r} a \Phi_{2}$ to 
within 0.001 at the half-power point of $f_{r n}$ (see Fig. B1d). If $f_{r n}$ is not symmetric, we use the half-power point on the more slowly decreasing side. Now $\Phi=\Phi_{1}+a \Phi_{2}$ can be recovered as shown in Fig. B1f.

It is important to note that no data are plotted in Fig. 2 between about $\Delta \varphi_{D r} \doteq-100^{\circ}$ and $\varphi_{D r} \doteq+100^{\circ}$. This is due to the $130^{\circ}$ width of the windowing function, $W$. Where no symbols are shown, the residual function $f_{r}$ does not exceed 0.15 and is often double peaked so $\Delta \varphi_{D r}$ cannot be determined. The exact angle at which this occurs depends on $\beta_{1}$. Therefore, this method cannot separate two spreading functions whose mean directions are closer together than this minimum angular separation. This applies only to wave trains of the same frequency, of course.

\section{REFERENCES}

Benoit, M., 1994: Extensive comparison of directional wave analysis methods from gauge array data. Ocean Wave Measurement and Analysis, American Society of Civil Engineers, 740-754.

Donelan, M. A., 2017: Frequency-direction spectra of wind-generated gravity waves. Encyclopedia of Marine and Offshore Engineering, Wiley, https://doi.org/10.1002/9781118476406.EMOE079.
— J. Hamilton, and W. H. Hui, 1985: Directional spectra of wind-generated waves. Philos. Trans. Roy. Soc. London, 315, 509-562, https://doi.org/10.1098/rsta.1985.0054.

— W. M. Drennan, and A. K. Magnusson, 1996: Nonstationary analysis of the directional properties of propagating waves. J. Phys. Oceanogr., 26, 1901-1914, https://doi.org/10.1175/ 1520-0485(1996)026<1901:NAOTDP $>2.0 . C O ; 2$.

_ A. Babanin, E. Sanina, and D. Chalikov, 2015: A comparison of methods for estimating directional spectra of surface waves. J. Geophys. Res. Oceans, 120, 5040-5053, https://doi.org/10.1002/ 2015JC010808.

Earle, M. D., K. E. Steele, and D. W. C. Wang, 1999: Use of advanced directional wave spectra analysis methods. Ocean Eng., 26, 14211434, https://doi.org/10.1016/S0029-8018(99)00010-4.

Hwang, P., D. W. Wang, W. E. Rogers, and W. B. Krabill, 2015: Bimodal directional propagation of wind-generated ocean surface waves. ResearchGate, https://doi.org/10.13140/RG.2.1.4591.5689.

Kuik, A. J., G. Ph. van Vledder, and L. H. Holthuijsen, 1988: A method for the routine analysis of pitch-and-roll buoy wave data. J. Phys. Oceanogr., 18, 1020-1034, https://doi.org/10.1175/ 1520-0485(1988)018<1020:AMFTRA > 2.0.CO;2.

Longuet-Higgins, M. S., D. E. Cartwright, and N. D. Smith, 1963: Observations of the directional spectrum of sea waves using the motions of a floating buoy. Ocean Wave Spectra, PrenticeHall, 111-136.

Steele, K. E., C.-C. Teng, and D. W. C. Wang, 1992: Wave direction measurements using pitch-roll buoys. Ocean Eng., 19, 349375, https://doi.org/10.1016/0029-8018(92)90035-3. 